\title{
Power division ratio on multi-hop WPT considering coupling of the receiver with two couplers
}

\author{
Akiro Shimada $^{1 a)}$, Shinsuke Ohtake ${ }^{1}$, Yuichi Miyaji ${ }^{2}$, \\ Hideyuki Uehara ${ }^{2}$, and Takashi Ohira ${ }^{2}$ \\ ${ }^{1}$ Graduate Program of Electrical and Electronic Information Engineering \\ ${ }^{2}$ Dept. of Electrical and Electronic Information Engineering, Toyohashi University \\ of Technology \\ a)shimada@comm.ee.tut.ac.jp
}

\begin{abstract}
We derive theoretically the power division ratio (PDR) on multi-hop wireless power transfer (WPT) to two receivers coupling with two couplers. The derived expression suggests that the PDR can be controlled by adjusting the load resistance. However, the control range of PDR by adjusting the load resistance of one receiver is limited when a hop count to that receiver is odd number. Therefore we show that the PDR can be controlled by another two methods; control of load resistances of each receiver and control of the load impedance of one receiver. These two methods can control the PDR within coaxial misalignment around the radius of the coupler.
\end{abstract}

Keywords: wireless power transfer, magnetic resonance, multiple receivers, power division, multi-hop

Classification: Electromagnetic theory

\section{References}

[1] B. L. Cannon, J. F. Hoburg, D. D. Stancil and S. C. Goldstein: IEEE Trans. Power Electron. 24 [7] (2010) 1819.

[2] T. Imura and Y. Hori: INTELEC (2011) 1.

[3] K. E. Koh, T. C. Beh, T. Imura and Y. Hori: WAMICON (2012) 1.

[4] K. E. Koh, T. C. Beh, T. Imura and Y. Hori: ICEMS (2012) 1.

[5] A. Shimada, Y. Ito, H. Uehara and T. Ohira: IWPTc (2013).

\section{Introduction}

Wireless power transfer (WPT) via magnetic resonance to mobile devices has got much attention. We often use more than one mobile device such as mobile phones and laptops. Hence, WPT to multiple mobile devices at the same time is required. Magnetic resonance enables power transfer to multiple receivers by using the same resonance frequency on a transmitter 
and receivers $[1,2]$. It is necessary to divide electric power to mobile devices appropriately because of the difference in the rated power of mobile devices (e.g. mobile phones and laptops). To solve this problem, a power division methodology by adjusting road impedance has been proposed [3]. Moreover, power division on multi-hop WPT to receivers coupling with one coupler has been considered to expand a power transfer range [4, 5]. However, those studies have not discussed coupling of receiver with multi-coupler. In this letter, we consider an effect of the coupling with two couplers on the power division ratio (PDR), and theoretically derive the PDR in such a case. Moreover the derived expression suggests that the PDR can be controlled by adjusting the load impedance, and then we show its propriety by an electromagnetic simulation.

\section{Effect of coupling of the receiver with two couplers on the PDR}

Here we discuss an effect of coupling of receivers with two couplers on the PDR. First, multi-hop WPT to one receiver coupling with two couplers as shown in Fig. 1 is considered. We assume that in a multi-hop WPT the transmitter and intermediate couplers are arranged at constant intervals and parasitic resistance and couplings between nonadjacent couplers are ignored. In this multi-hop WPT, the hop count takes an odd or even number [5]. Hence, equivalent circuits of every even-hopped and odd-hopped WPT to one receiver coupling with two couplers are shown in Fig. 2, where $M_{12}^{\prime}$ is mutual inductance of receiver 2 and $(n-2)$ th coupler and $M_{12}$ is mutual inductance of receiver 2 and $(n-1)$ th coupler. $M$ is mutual inductance of couplers that are arranged on constant intervals. We can get the Eqs. (1) and (2) by using Kirchhoff's voltage laws on Figs. 2.

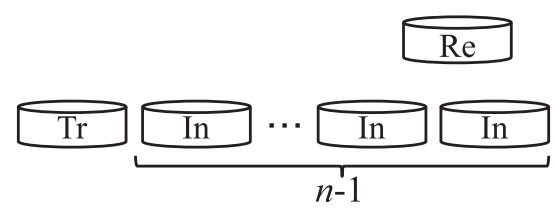

Fig. 1. Multi-hop WPT to one receiver coupling with two couplers.

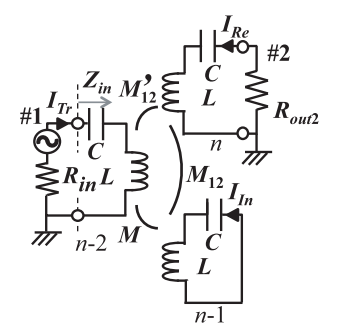

(a)

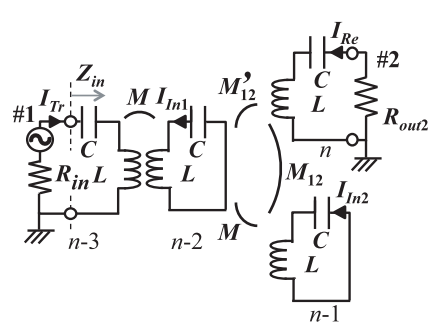

(b) (c) IEICE 2014

DOI: $10.1587 /$ elex.11.20140034

Received January 14, 2014

Accepted January 21, 2014

Publicized February 17, 2014

Copyedited March 10, 2014 


$$
\begin{aligned}
& {\left[\begin{array}{c}
V_{\text {in }} \\
0 \\
0
\end{array}\right]=\left[\begin{array}{ccc}
R_{\text {in }}+a & j \omega M & j \omega M_{12}^{\prime} \\
j \omega M & a & j \omega M_{12} \\
j \omega M_{12}^{\prime} & j \omega M_{12} & R_{\text {out } 2}+a
\end{array}\right]\left[\begin{array}{c}
I_{T r} \\
I_{I n} \\
I_{R e}
\end{array}\right],(n: \text { even }) .} \\
& a=j \omega L-j \frac{1}{\omega C} . \\
& {\left[\begin{array}{c}
V_{\text {in }} \\
0 \\
0 \\
0
\end{array}\right]=\left[\begin{array}{cccc}
R_{\text {in }}+a & j \omega M & 0 & 0 \\
j \omega M & a & j \omega M & j \omega M_{12}^{\prime} \\
0 & j \omega M & a & j \omega M_{12} \\
0 & j \omega M_{12}^{\prime} & j \omega M_{12} & R_{\text {out } 2}+a
\end{array}\right]\left[\begin{array}{c}
I_{T r} \\
I_{\text {In } 1} \\
I_{\text {In } 2} \\
I_{R e}
\end{array}\right],(n: \text { odd }) .}
\end{aligned}
$$

From Eqs. (1) and (2), receiver to transmitter current ratio $\left|I_{R e} / I_{T r}\right|$ and input impedance $Z_{\text {in }}$ are derived as

$$
\begin{aligned}
& \left|\frac{I_{R e}}{I_{T r}}\right|=\left\{\begin{array}{l}
\frac{M}{M_{12}},(n: \text { even }) \\
\mid \frac{\omega_{0} M_{12}}{R_{\text {out } 2}-j 2 \omega_{0} \frac{M_{12} M_{12}^{\prime}}{M} \mid,}(n: \text { odd }) .
\end{array}\right. \\
& Z_{\text {in }}=\left\{\begin{array}{l}
\frac{M^{2}}{M_{12}^{2}} R_{\text {out } 2}-j 2 \omega_{0} \frac{M M_{12}^{\prime}}{M_{12}},(n: \text { even }) \\
\frac{\omega_{0}^{2} M^{2}}{\frac{M^{2}}{M_{12}^{2}} R_{\text {out } 2}-j 2 \omega_{0} \frac{M M_{12}^{\prime}}{M_{12}},},(n: \text { odd }) .
\end{array}\right.
\end{aligned}
$$

We can find from Eq. (3) that $M_{12}^{\prime}$ gives an effect on $\left|I_{R e} / I_{T r}\right|$ only when hop count $n$ is odd. On the other hand, the input impedance is changed by $M_{12}^{\prime}$ independent of the parity of the hop count.

Next, we derive the PDR on multi-hop WPT to two receivers coupling with two couplers as shown in Fig. 3 (a). An equivalent circuit of Fig. 3 (a) is shown in Fig. 3 (b). The PDR is defined as the ratio of received power of receiver 2 and receiver 3 , and expressed as

$$
\eta_{21}: \eta_{31}=\left|I_{\text {Re2 }}\right|^{2} R_{\text {out } 2}:\left|I_{\text {Re3 }}\right|^{2} R_{\text {out } 3} .
$$

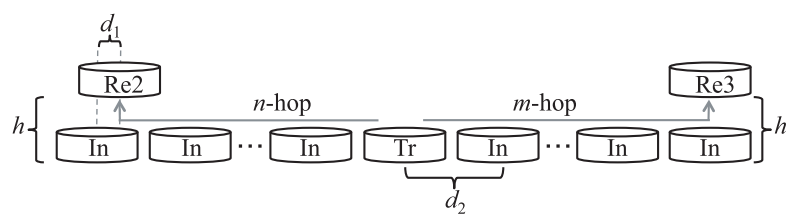

(a)

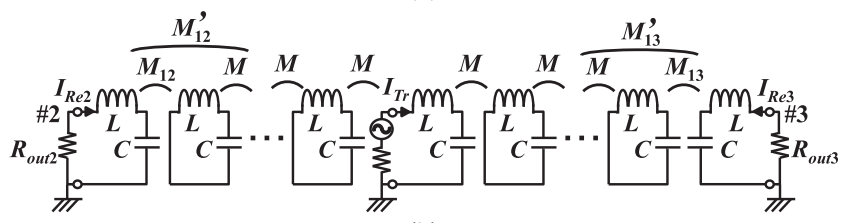

(b) 
The PDR is given by substituting each receiver's current to Eq. (5). The current of each receiver can be calculated by Eq. (3), and the derived PDR is expressed as Eq. (6). It is found from Eq. (6) that the PDR is affected by coupling with two couplers when a hop count to at least one receiver is odd number.

According to the study above, we suggest two impedance control methods based on the derived PDR. The first method controls load resistances of each receiver. The second one controls the load impedance of one receiver. Assuming two cases that $n, m=$ odd and $n, m=$ even. Given $\eta_{21}: \eta_{31}=R_{21}$ : $R_{31}$, load resistance $R_{\text {out } 2}$ is calculated by Eq. (7)

$$
\begin{aligned}
& \eta_{21}: \eta_{31}= \\
& \left\{\begin{array}{l}
\left|\frac{\omega_{0} M_{12}}{R_{\text {out } 2}-j 2 \omega_{0} \frac{M_{12} M_{12}^{\prime}}{M}}\right|^{2} R_{\text {out } 2}:\left|\frac{\omega_{0} M_{13}}{R_{\text {out } 3}-j 2 \omega_{0} \frac{M_{13} M_{13}^{\prime}}{M}}\right|^{2} R_{\text {out } 3},(n, m: \text { odd }) \\
\frac{R_{\text {out } 2}}{M_{12}^{2}}: \frac{R_{\text {out } 3}}{M_{13}^{2}},(n, m: \text { even }) \\
\left|\frac{\omega_{0} M_{12}}{R_{\text {out } 2}-j 2 \omega_{0} \frac{M_{12} M_{12}^{\prime}}{M}}\right|^{2} R_{\text {out } 2}: \frac{M^{2} R_{\text {out } 3}}{M_{13}^{2}},(n: \text { odd }, m: \text { even }) \\
\frac{M^{2} R_{\text {out } 2}}{M_{12}^{2}}:\left|\frac{\omega_{0} M_{13}}{R_{\text {out } 3}-j 2 \omega_{0} \frac{M_{13} M_{13}^{\prime}}{M}}\right|^{2} R_{\text {out } 3},(n: \text { even, } m \text { :odd }) .
\end{array}\right.
\end{aligned}
$$

$$
\begin{aligned}
& R_{\text {out } 2}= \\
& \left\{\begin{array}{l}
\frac{\omega_{0}^{2} M_{12}^{2} R_{31}+\omega_{0} M_{12} \sqrt{\omega_{0}^{2} M_{12}^{2} R_{31}^{2}-16 a^{2} R_{21}^{2} \frac{M_{12}^{\prime 2}}{M^{2}} R_{\text {out } 3}^{2}}}{2 R_{21} a R_{\text {out } 3}},(m, n: \text { odd }) \\
\frac{R_{21} M_{12}^{2}}{R_{31} M_{13}^{2}} R_{\text {out } 3},(m, n: \text { even }),
\end{array}\right.
\end{aligned}
$$

where, $\quad a=\frac{\omega_{0}^{2} M_{13}^{2}}{R_{\text {out } 3}^{2}+4 \omega_{0}^{2} M_{13}^{2} M_{13}^{\prime 2} / M^{2}}$.

This equation indicates that we can control the PDR by adjusting load resistance $R_{\text {out } 2}$. However, if $R_{\text {out } 2}$ is complex number, the PDR cannot be controlled by load resistance $R_{\text {out } 2}$. In this case, it is one possible method to adjust load resistance $R_{\text {out } 3}$ too so that $R_{\text {out } 2}$ takes real number. The other method is to add reactance in series with load resistance $R_{\text {out } 3}$ to cancel the imaginary part in Eq. (3). When the imaginary part is perfectly canceled by the added reactance, $R_{\text {out } 2}$ is rewritten as follows.

$$
R_{\text {out } 2}=\frac{R_{31} M_{12}^{2}}{R_{21} M_{13}^{2}} R_{\text {out } 3},(m, n: \text { odd }) .
$$




\section{Simulation}

We validate the derived PDR by electromagnetic simulation of Fig. 3 (a). WIPL-D is used as a electromagnetic simulator. In cases of hop counts $n, m=3$ and $n, m=2$, we attempt to divide power into two receivers with PDR of $\eta_{21}: \eta_{31}=6: 4$ by calculating Eqs. (7) and (8). To calculate Eqs. (7) and (8), the mutual inductances and parameters of the coupler have measured by electromagnetic simulations. The configuration for the simulation in case of hop counts $n, m=2$ is shown in Fig. 4. Simulation parameters are shown in Table I. The simulation results of the PDR of receiver 2 are shown in Figs. 5 and 6 , where $R_{21}$ is defined by $\eta_{21} /\left(\eta_{21}+\eta_{31}\right) \times 100$ [\%], and $d_{1}$ express coaxial misalignment. We intend to control the $R_{21}$ on $60 \%$. In Fig. 5, the control range of the first method is expanded as increasing $R_{\text {out } 3}$. It is because $R_{\text {out } 2}$ in Eq. (7) is likely to complex number with small $R_{\text {out } 3}$ when the couplings of the receiver with two couplers become strong. The control range of the second one is larger than first one with same $R_{\text {out } 3}$. This reason is that the added reactance cancels the effect of the coupling of the receiver with two couplers. Fig. 6 shows only the result of the first method because the coupling of the receiver with two couplers does not give any effects on the PDR. Therefore, the reactance is not needed to cancel the effect of the coupling with two couplers. We can control the PDR without

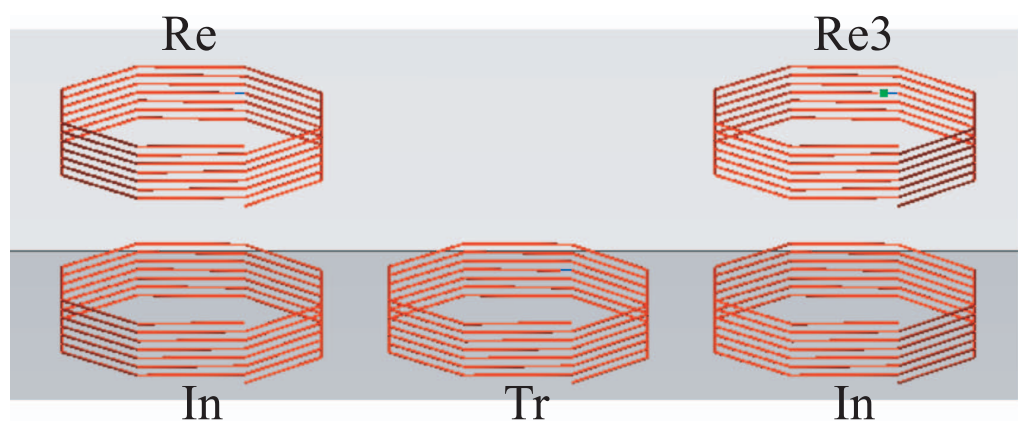

Fig. 4. Configuration of simulation for multi-hop WPT to two receivers $(n, m=2)$.

Table I. Simulation parameters.

\begin{tabular}{l|l}
\hline Symbol & Value \\
\hline Structure of the coupler & 8 -cornered helical \\
\hline Diameter of the coupler & $300 \mathrm{~mm}$ \\
\hline Turns of the coupler & 7 \\
\hline Height of the coupler & $78 \mathrm{~mm}$ \\
\hline Lead radius & $1.5 \mathrm{~mm}$ \\
\hline Material of lead & Perfect conductor \\
\hline Resonant frequency & $14.3 \mathrm{MHz}$ \\
\hline Distance of coaxial misalignment $d_{1}$ & $0 \sim 400 \mathrm{~mm}$ \\
\hline Distance between intermediate couplers $d_{2}$ & $350 \mathrm{~mm}$ \\
\hline Height between the coupler and receiver $h$ & $200 \mathrm{~mm}$ \\
\hline
\end{tabular}




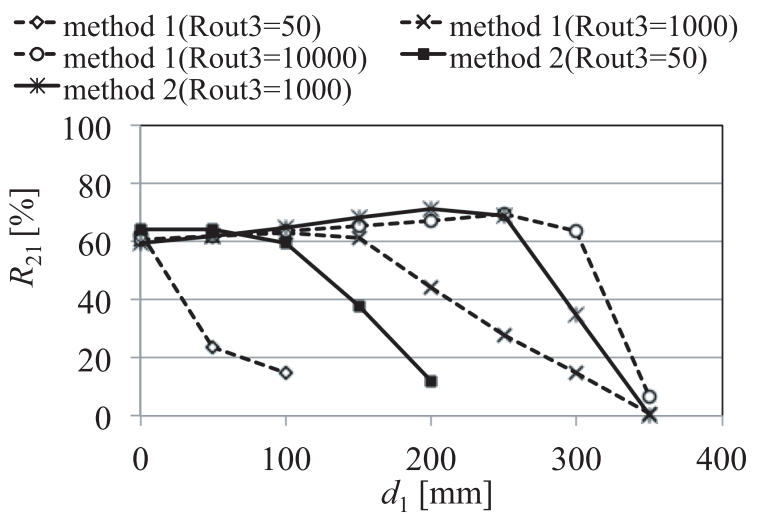

Fig. 5. The PDR of the receiver 2 in odd-hop.

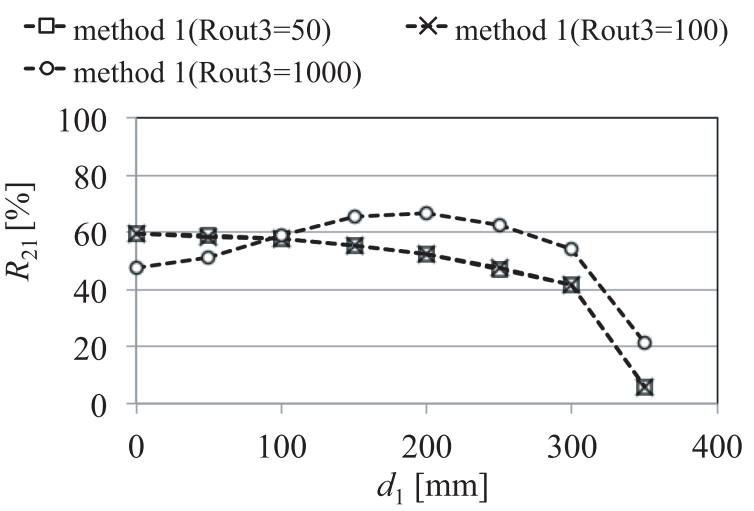

Fig. 6. The PDR of the receiver 2 in even-hop.

considering the effect of the coupling with two couplers in even-hop. It is found from Figs. 5 and 6 that the difference from $R_{21}=60 \%$ is less than 9 points when coaxial misalignment $d_{1}$ is smaller than the radius of the coupler in both methods. Therefore, these two methods can control the PDR even if coaxial misalignment is around the radius of the coupler. However, both methods cannot control the PDR if coaxial misalignment $d_{1}$ is larger than the diameter of the coupler. Those difference and uncontrollable state come from the assumptions when we derived Eq. (7), that is, ignored couplings between the receiver, transmitter, and nonadjacent couplers.

\section{Conclusion}

In this letter, we have derived theoretically the PDR on multi-hop WPT to two receivers coupling with two couplers. The derived PDR expression has suggested that the effect of the coupling of the receiver with two receivers on the PDR limits the control range of the PDR by adjusting load resistance $R_{\text {out } 2}$ when a hop count to one receiver is an odd number. Hence we have showed that the PDR can be controlled by two impedance control methods to get the intended PDR. One is control of load resistances of each receiver. The second one is control of the load impedance of one receiver. These two methods can control the PDR by coaxial misalignment around the radius of the coupler. 